\section{Absenteísmo-doença entre profissionais de enfermagem da rede básica do SUS Campinas*}

\section{Sick leaves among nursing professionals in basic healthcare centers of the Brazilian National Unified Healthcare System - SUS - in the city of Campinas}

\author{
Gilson Gehring Junior ${ }^{1}$ \\ Heleno Rodrigues Corrêa Filho \\ Joana D'Arc Vieira Neto $^{3}$ \\ Nely Alves Ferreira ${ }^{4}$ \\ Simone V.R. Vieira ${ }^{5}$ \\ ${ }^{1}$ Hospital.Estadual Sumaré - Sumaré, SP \\ ${ }^{2}$ Departamento de Medicina Preventiva e Social - FCM - Unicamp, Campinas, \\ $\mathrm{SP}$ \\ ${ }^{3}$ Farmácia de Alto Custo da DIR XII - Campinas, SP \\ ${ }^{4}$ Unidade de Saúde do Trabalhador do Hosp. Dr. Mario Gatti - Campinas, SP \\ ${ }^{5}$ Pronto Atendimento Ouro Verde - Campinas, SP
}

\section{Resumo}

O objetivo deste trabalho é analisar índices de absenteísmo-doença entre profissionais de enfermagem de 16 Unidades Básicas de Saúde do Município de Campinas durante o ano de 2002. Foram obtidos junto à seção de Recursos Humanos Descentralizados dados de assiduidade de 311 trabalhadores de enfermagem de Centros de Saúde dos Distrito Norte e Leste do Município de Campinas, e calculados os índices de freqüência, gravidade e proporção de tempo perdido. As variáveis disponibilizadas foram tempo de trabalho na função, categoria funcional, sexo e carga horária semanal. Dados de pesquisas realizadas sobre absenteísmo com pessoal de hospital e do ramo industrial e empresarial foram utilizados para a análise e comparação com o presente estudo. A função de técnico ou auxiliar de enfermagem representou a maioria da população $(82,6 \%)$, a jornada de trabalho de 36 horas semanais foi realizada por $90,6 \%$ dos pesquisados e $93,5 \%$ eram do sexo feminino. $O$ índice de freqüência de absenteísmo foi de 2,88 afastamentos iniciados por empregado/ ano, o de gravidade foi de 12,27 dias perdidos por empregado/ano, e a proporção de tempo perdido foi de $5,56 \%$ ao ano. Os enfermeiros tiveram índice de freqüência menor que o de auxiliares e técnicos. Os índices encontrados neste trabalho foram maiores do que a maioria dos estudos realizados com profissionais de hospitais. A fim de minimizar o fenômeno como reflexo do impacto das condições desfavoráveis para a saúde do trabalhador, propõe-se diminuir da exposição às cargas de trabalho e melhorar a política de saúde do trabalhador para os servidores da categoria.

Palavras-chave: Absenteísmo. Epidemiologia. Saúde do trabalhador. Condições de trabalho.

'Trabalho resultante de Projeto de Iniciação Científica Finaciado pelo CNPq. Processo número 105817/2001-5.

Correspondência: Gilson Gehring Junior. R. Bethânia, 575 - Hortolândia, SP, CEP 13184-030.

E-mail: gehringjunior@universiabrasil.net 
Abstract

This report aims to analyze sick leave rates among healthcare professionals from 16 Basic Healthcare Units of the Brazilian National Unified Healthcare System (SUS) in the Municipality of Campinas during 2002. Data on worked days and sick leaves of 311 nurses and nursing aides working at Healthcare Centers of the North and East regions of the municipality of Campinas were obtained at the central administration, in order to calculate the frequency, severity and proportion of lost working time. Variables used were time on the job, job title, gender and weekly hour load. Results of the present study were compared with other published reports on sick leave rates in hospitals, manufacturing, and service settings. Most of the studied population comprised nursing aides and other healthcare technicians (82.6\%), $90.6 \%$ had a weekly work load of 36 hours, and $93.5 \%$ of the group were women. Absenteeism frequency rate was of 2.88 new sick leaves per employee/year. The severity index was of 12.27 lost working days per employee/ year, and the proportion of work time lost was $5.56 \%$ a year. Registered Nurses had lower frequency rates than nursing aides and technicians. Findings pointed to higher indexes and rates when compared with hospital data from other publications. A proposal is presented to minimize this phenomenon that results from the impact of conditions that are unfavorable to workers' health, through the reduction of the workload and the improvement of public health policy for this group of workers.

Keywords: Absenteeism. Epidemiology. Occupational health. Working conditions

\section{Introdução}

Ao se trabalhar com absenteísmo convém esclarecer a abordagem que é dada ao tema. A palavra "absenteísmo" tem sua origem no francês (absentéisme) e significa falta de assiduidade ao trabalho ou a outras obrigações sociais ${ }^{1}$. Podem ser diversas as causas desencadeantes da abstenção do trabalho: problemas de saúde ou doenças, doenças ou acidentes do trabalho, problemas de saúde em pessoas da família, gestação e parto, casamento (licença gala), entre outros. Podem ocorrer ainda faltas que não são justificadas legalmente, conhecidas como faltas injustificadas $^{2-4}$. Nessa perspectiva, entende-se que absenteísmo-doença se refere ao não comparecimento ao trabalho por motivo de doença ou problema de saúde, relacionado ou não ao trabalho.

Em hospitais o absenteísmo tem recebido destaque nos últimos anos, porém não foram encontrados trabalhos abordando o assunto na rede básica de saúde. Nos estudos realizados em hospitais, entre todas as causas, os agravos à saúde foram os maiores causadores de afastamentos do trabalho ${ }^{2,4-7}$.

A periculosidade e a insalubridade do trabalho de enfermagem são caracterizadas pela exposição a microrganismos patogênicos (risco biológico), a radiações (risco físico) e a substâncias tóxicas (risco químico), entre outros agentes. Já o caráter penoso deste trabalho é influenciado pela grande quantidade de atividades que exigem esforço físico, por más condições do ambiente de trabalho e por tensões nas relações interpessoais ${ }^{8}$. Nesse contexto, o ambiente de trabalho na enfermagem pode ser um agente catalisador do processo de desgaste do trabalhador, culminando em doenças'.

As características de gênero tem mostrado importante papel no desencadeamento do absenteísmo ${ }^{11}$. Embora as pesquisas apontem para as mulheres como tendo índices de absenteísmo maiores que os homens, é reconhecida a necessidade 
de mais pesquisas para elucidar o assunto ${ }^{10-12}$. Os fatores relacionados com o absenteísmo feminino vão desde a necessidade de cuidado dos filhos e das tarefas domésticas até a maior suscetibilidade ao estresse e a problemas de saúde. Mesmo que tenha apenas um emprego, é comum a mulher enfrentar a dupla-jornada, representada pela associação do trabalho "fora de casa" com o trabalho doméstico ${ }^{11,13,14}$.

A ausência de funcionários no trabalho é influenciada por inúmeros fatores, intrínsecos ou extrínsecos ao trabalho, que atuam sobre a pessoa tornando o absenteísmo um problema de alta complexidade. Como conseqüências do fenômeno verifica-se a desorganização do trabalho em equipe, sobrecarga de trabalho, insatisfação dos trabalhadores, queda na qualidade e quantidade do trabalho realizado. Dessa forma são afetados os interesses da instituição e os direitos do trabalhador, bem como a relação de trabalho entre ambos ${ }^{2,3,6,11,15-18}$.

Quando o absenteísmo é abordado a partir da perspectiva dos empregadores, busca-se a redução do absenteísmo por meio do aumento de medidas de controle e com pouco ou nenhum enfoque para as ações de prevenção e promoção da saú$\mathrm{de}^{19}$. No presente trabalho, a posição que se pretende tomar diante ao absenteísmo é defender a saúde do trabalhador, utilizando os dados obtidos como subsídio na discussão da melhoria das condições de trabalho, na promoção da saúde e na prevenção de agravos à saúde.

O objetivo deste trabalho foi analisar índices de absenteísmo entre profissionais de enfermagem de 16 Unidades Básicas de Saúde do Município de Campinas durante o ano de 2002, na busca de compreender as causas do problema, na perspectiva da promoção e defesa da saúde do trabalhador.

\section{Métodos}

\section{População Estudada}

A rede básica de saúde do município de Campinas conta com 45 Centros de Saú- de (CSs), responsáveis pela atenção à saúde em nível primário. A população deste estudo é constituída por todos os enfermeiros, auxiliares e técnicos de enfermagem que trabalharam no ano de 2002 nos 16 Centros de Saúde pertencentes aos Distritos Norte e Leste do município.

\section{Coleta de Dados}

Os dados foram obtidos junto à seção de Recursos Humanos Descentralizados (RHD) do Distrito Norte e Leste, após aprovação do protocolo pelo Comitê de Ética em Pesquisa da Faculdade de Ciências Médicas da Unicamp. Foram consultados formulários de controle de assiduidade dos 311 profissionais durante todo o ano de 2002. Por se tratar de dados institucionais, não houve perdas. Os autores declararam estar trabalhando sem conflitos de interesse.

Foi elaborado pelos autores um formulário simplificado semelhante ao dos RHD. Compondo o formulário do RHD existia um quadro contendo todos os dias e meses do ano de 2002, no qual os tipos de ausência eram marcados, inclusive as férias e licenças-prêmio. Entretanto, dias como sábados, domingos e feriados não eram especificados naquele formulário. No formulário simplificado esses dias foram incluídos, o que permitiu conhecer os dias previstos para serem trabalhados, os dias realmente trabalhados e os dias perdidos por licença para tratamento de saúde (LTS). Fizeram parte do formulário dados como sexo, função (enfermeiro ou auxiliar/técnico de enfermagem), carga horária (30 ou 36 horas semanais) e tempo de trabalho pela Secretaria de Saúde do Município de Campinas (SSMC) na respectiva função. O departamento de RHD não dispunha da variável idade. Os dados foram inseridos em base de dados e analisados com o programa EpiInfo versão 6.04d.

O trabalho de Silva (1983) estimou as ausências por motivo de férias reduzindo $1 / 12$ do tempo previsto de trabalho. No presente trabalho não foi feita estimativa, uma vez que o formulário dos RHD dispunha dos 
cálculos exatos dos dias que cada profissional retirou de férias ou licença prêmio.

\section{Análise dos Dados}

A partir dos dados obtidos foram calculados os índices de freqüência, gravidade e proporção de tempo perdido. Em trabalhos de revisão posteriores a 1993 não foram encontradas referências sobre fundamentos da utilização de índices de freqüência e gravidade $^{20}$. Assim, foram utilizadas para os cálculos referências anteriores.

O índice de freqüência de absenteísmo foi calculado conforme apresentado por Corrêa Filho $(1993)^{21}$ e Mendes (1980) ${ }^{22}$, dividindo-se o número total de afastamentos iniciados no ano de 2002 pelo número total de empregados que trabalharam nesse período.

FREQÜÊNCIA $=\frac{\begin{array}{c}\text { Número de afastamentos devido } \\ \text { a LTS iniciados em } 2002\end{array}}{\text { Número total de empregados em } 2002}$

O índice de gravidade de absenteísmo segue o recomendado por Mendes (1980) ${ }^{22}$ como a razão entre o número de dias perdidos devido a LTS e o número médio de empregados. O tempo perdido é calculado em dias, a fim de possibilitar comparações com outros trabalhos.

GRAVIDADE $=\frac{\text { Tempo perdido por LTS }(\text { em dias ou horas) }}{\text { Número total de empregados em } 2002}$

Para o cálculo da proporção de tempo perdido por LTS seguiu-se o proposto por Mendes (1980) ${ }^{22}$; entretanto, os dias foram convertidos em horas.

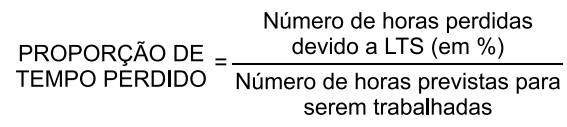

\section{Resultados}

Dos 311 profissionais pesquisados, 257 (82,6\%) são auxiliares ou técnicos de enfermagem e 54 (17,4\%) enfermeiros. Quanto ao gênero, $291(93,56 \%)$ são do sexo fe- minino e $20(6,43 \%)$ do sexo masculino. Em relação à jornada de trabalho, encontrouse que $9,4 \%$ e $90,6 \%$, respectivamente, têm jornada de 30 e 36 horas semanais. Não se tem conhecimento ou registro de prêmios em dinheiro para os que não faltam ao trabalho. É permitida a ausência sem justificativa ao trabalho por um dia a cada dois meses. É possível que trabalhadores usem essa falta para cuidados à própria saúde com subnotificação de faltas.

A Tabela 1 mostra a porcentagem de trabalhadores de enfermagem segundo a quantidade de meses que exerceram a respectiva função na SSMC até 2002. Com relação a esse tempo de trabalho, a maior parte $(56,3 \%)$ deles está na faixa de 4 a 8 anos. Uma vez que a SSMC é composta por CSs e hospitais, não é possível afirmar que todo esse tempo foi trabalhado em CSs.

Tabela 1 - Distribuição da porcentagem de funcionários de acordo com a quantidade de meses que exerceu a função na Secretaria de Saúde de Campinas até 2002.

Table 1 - Percentage distribution according to the number of months in the job at Campinas Health Department up to 2002.

\begin{tabular}{lc}
\hline Tempo de trabalho na função $\%$ & \\
\hline Até 24 meses & 12,2 \\
$25-48$ meses & 7,4 \\
$49-72$ meses & 37,6 \\
$73-96$ meses & 18,7 \\
$97-120$ meses & 2,2 \\
$121-180$ meses & 16,4 \\
Mais que 181 meses & 5,5 \\
Total & 100,0 \\
\hline
\end{tabular}

Na população estudada, encontrou-se índice de freqüência de 2,88 afastamentos iniciados por empregado/ano, gravidade de 12,27 dias perdidos por empregado/ ano. A proporção de tempo perdido foi de $5,56 \%$ ao ano (Tabela 2).

Apesar do grupo de auxiliares e técnicos de enfermagem apresentar índices de freqüência de afastamentos por LTS maior que o grupo de enfermeiros, ambos apresentaram índices de gravidade e proporção 
Tabela 2 - Distribuição da população, índices de freqüência, gravidade e proporção de tempo perdido de acordo com função e sexo durante 2002.

Table 2 - Distribution of population, frequency and severity indexes and proportion of work time lost according to job title and gender during 2002.

\begin{tabular}{|c|c|c|c|c|}
\hline Variável & $\begin{array}{l}\text { População } \\
\text { por Grupo }\end{array}$ & $\begin{array}{l}\text { Gravidade } \\
\text { (dias perdidos } \\
\text { devido a LTS/ } \\
\text { empregado) }\left(^{*}\right)\end{array}$ & $\begin{array}{c}\text { Freqüência } \\
\text { (afastamentos } \\
\text { iniciados/ } \\
\text { empregado) }\left(^{*}\right)\end{array}$ & $\begin{array}{c}\text { Proporção de } \\
\text { Tempo Perdido } \\
\text { (horas perdidas devido } \\
\text { a LTS/ horas previstas } \\
\text { de trabalho) }\left(^{*}\right)\end{array}$ \\
\hline \multicolumn{5}{|l|}{ Categoria Funcional } \\
\hline Enfermeiro & 54 & $12,70(4,0-21,3)$ & $1,70(1,1-2,3)$ & $5,6 \%(5,5-5,6 \%)$ \\
\hline $\begin{array}{l}\text { Técnico/ Auxiliar de } \\
\text { enfermagem } \\
\text { Sexo }\end{array}$ & 257 & $12,18(9,2-15,0)$ & $3,13(2,7-3,6)$ & $5,57 \%(5,50-5,64 \%)$ \\
\hline Masculino & 20 & $7,2(6,1-8,4)$ & $2,4(1,2-3,7)$ & $3,1(2,9-3,3)$ \\
\hline Feminino & 291 & $12,6(9,6-15,6)$ & $2,9(2,5-3,3)$ & $5,7(5,6-5,8)$ \\
\hline \multicolumn{5}{|l|}{ Total de Dias de } \\
\hline \multicolumn{5}{|c|}{ Afastamentos em 2002} \\
\hline $0-7$ & 196 & $1,7(1,4-2,0)$ & $1,2(1,0-1,4)$ & $0,80(0,77-0,84)$ \\
\hline 8 ou mais & 115 & $30,2(23,8-36,5)$ & $5,7(5,0-6,4)$ & $13,1(12,9-13,2)$ \\
\hline População Inteira & 311 & $12,27(9,5-15,1)$ & $2,88(2,5-3,3)$ & $5,56 \%(5,5-5,6)$ \\
\hline
\end{tabular}

(*) Intervalos de Confiança para 95\%./ 95\% Confidence intervals.

de tempo perdido semelhantes, evidenciando maior duração dos afastamentos dos enfermeiros em relação aos de auxiliares e técnicos. Com relação ao gênero, as mulheres tiveram proporção de tempo perdido e índice de gravidade de absenteísmo maiores que os homens (Tabela 2).

A população foi dividida em dois grupos conforme o total de dias de afastamentos em 2002. Conforme a Tabela 2, os índices de freqüência, gravidade e proporção de tempo perdido daqueles que tiveram 7 ou menos dias de licença foram menores que o outro grupo. A proporção de tempo perdido desses grupos mostra que as maiores perdas de tempo de trabalho refere-se aos trabalhadores que têm longos períodos de afastamento.

Não foi encontrada relação do tempo de trabalho na função pela SSMC com o número de LTS iniciadas e o total de tempo perdido por LTS. Houve ainda 29 pessoas com 143 faltas injustificadas no ano, todas elas entre auxiliares de enfermagem

\section{Discussão}

O grupo dos enfermeiros possui afastamentos de maior duração que o grupo dos auxiliares e técnicos. Assim, o fato de os afastamentos de curta duração não serem freqüentes entre enfermeiros pode ter relação com a divisão do trabalho nos Centros de Saúde. Considerando que o número de enfermeiros nesses locais é menor que o de auxiliares e técnicos de enfermagem, e que aquela categoria é responsável pelo trabalho de enfermagem na unidade, os enfermeiros podem estar optando por trabalhar doentes em vez de se afastar por curtos intervalos e deixar a equipe sem uma pessoa de referência.

É possível que uma maior proporção de tempo perdido no gênero feminino esteja relacionada à dupla jornada, que, na maioria das vezes, não é caracterizada por dois empregos, mas pelo trabalho e afazeres domésticos que as mulheres realizam após a jornada normal. Caracterís- 
ticas de morbidade feminina, como os distúrbios ligados ao ciclo hormonal, também são determinantes do absenteísmo entre mulheres ${ }^{14}$.

Quando considerado o total de dias de afastamentos e o número de afastamentos iniciados, os maiores índices estiveram entre trabalhadores que possuíram oito ou mais dias de afastamento. Esse perfil de afastamento se assemelha ao dos portadores de doenças crônicas, os quais, após determinado período de licença, freqüentemente necessitam permanecer ausentes por não estar condições de retornar ao trabalho.

Diante da necessidade de comparação deste estudo com a literatura, e da melhor visualização dos dados, fez-se necessária a inclusão de uma tabela (Tabela 3) na discussão.

A literatura mostra que os índices de absenteísmo entre pessoal de enferma- gem são maiores que os de profissionais de outros ramos, como o industrial e o empresarial.

No atual trabalho foi constatado que o pessoal de enfermagem da rede básica apresentou índices de gravidade e freqüência de absenteísmo discretamente elevados, quando comparados à maioria dos estudos realizados em hospitais. Dos oito trabalhos encontrados para comparação, apenas dois exibem índices contidos no intervalo de confiança calculado para a rede básica.

O maior absenteísmo entre trabalhadores de enfermagem pode estar relacionado às cargas psíquicas enfrentadas no ambiente de trabalho, tanto em hospitais quanto na rede básica. Greco $(2001)^{26}$, analisando as cargas às quais o pessoal de rede básica é exposto, afirma que aquela a que ele mais está exposto é a psíquica. Esta é

Tabela 3 - Comparação dos dados do presente trabalho com os disponíveis na literatura.

Table 3 - Comparison with other published data.

\begin{tabular}{|c|c|c|c|c|}
\hline Trabalho & População Estudada & $\begin{array}{l}\text { Gravidade } \\
\text { (dias perdidos } \\
\text { devido a LTS/ } \\
\text { empregado) }\end{array}$ & $\begin{array}{l}\text { Freqüência } \\
\text { (afastamentos } \\
\text { iniciado s/ } \\
\text { empregado) }\end{array}$ & $\begin{array}{c}\text { Proporção de } \\
\text { Tempo Perdido } \\
\text { (horas perdidas } \\
\text { devido a LTS/ } \\
\text { horas previstas } \\
\text { de trabalho) }\end{array}$ \\
\hline Presente trabalho & $\begin{array}{l}\text { Pessoal de enfermagem de } \\
\text { atenção primária }(n=311)\end{array}$ & 12,3 & 2,9 & $5,9 \%$ \\
\hline Hamoui et al.(2005) ${ }^{27}$ & $\begin{array}{l}\text { Pessoal administrativo de } \\
\text { universidade pública } \\
\text { ( } \mathrm{n}=\text { variou de } 2151 \text { a 2259) }\end{array}$ & $\begin{array}{l}\text { Variou entre } \\
4,6 \text { e } 5,8\end{array}$ & $\begin{array}{l}\text { Variou entre * } \\
\quad 0,4^{*} \text { e } 0,5\end{array}$ & - \\
\hline Silva; Marziale $(2000)^{2}$ & $\begin{array}{l}\text { Pessoal de enfermagem de } \\
\text { hospital universitário }(n=199)\end{array}$ & $7,5^{*}$ & $2,5^{*}$ & - \\
\hline Echer et al. (1999) ${ }^{6}$ & $\begin{array}{l}\text { Pessoal de enfermagem de } \\
\text { hospital universitário }(n=211)\end{array}$ & $13,7^{*}$ & - & - \\
\hline \multirow[t]{2}{*}{ Saroja et al. (1999) ${ }^{23}$} & Agências não especificadas & $\begin{array}{l}\text { Variou entre } \\
2,4 \text { e } 3,8\end{array}$ & $\begin{array}{l}\text { Variou entre } \\
1,2 \text { e } 1,7\end{array}$ & $\begin{array}{l}\text { Variou entre } \\
1,9 \% \text { e } 2,6 \%\end{array}$ \\
\hline & $(n=$ variou entre 325 e 334$)$ & & & \\
\hline Robazi et al. $(1990)^{4}$ & $\begin{array}{l}\text { Pessoal de enfermagem de } \\
\text { hospital universitário }(n=20)\end{array}$ & $7,2^{*}$ & - & - \\
\hline Pines et al. (1985) $)^{12}$ & Pessoal de hospital $(n=3006)$ & 11,5 & 2,7 & - \\
\hline Quick; Lapertosa (1982) ${ }^{24}$ & $\begin{array}{l}\text { Trabalhadores de usina } \\
\text { siderúrgica }(n=10.112)\end{array}$ & 2,8 & 0,8 & - \\
\hline Meira $(1982)^{25}$ & Indústria química (" $n$ " não informado) & 7,8 & 1,2 & - \\
\hline
\end{tabular}

*Índices calculados a partir de dados indiretos obtidos nos trabalhos daqueles autores.

* Rates calculated or estimated based on indirect data from the authors' reports. 
representada pela insatisfação dos usuários, carência de recursos humanos, sobrecarga de trabalho e capacidade de atendimento menor do que a procura.

Há evidências de que os maiores índices de gravidade e freqüência de afastamentos referentes à população deste estudo sejam decorrentes direta ou indiretamente do sofrimento mental. Possíveis explicações para isso são: trabalho desagregado em pequenas equipes; coordenação de unidades trabalhando isoladas dos corpos de decisão política, administrativa e sanitária, ou trabalho solitário; trabalho assistencial exercido com baixa resolutividade em virtude de pouco suporte diagnóstico, demora dos resultados e meios escassos de tratamento na área básica. Apesar de ser encontrado na literatura que a relação com os usuários, a forma de organização do sistema de saúde e o trabalho em equipe são causas geradoras de sofrimento no trabalho ${ }^{28}$, são necessárias pesquisas futuras que procurem um modelo de causalidade.

Considerando as cargas peculiares ao exercício da profissão no contexto estudado como contribuintes ao desencadeamento do processo de adoecimento do profissional de enfermagem, sugere-se como intervenção a diminuição da exposição principalmente à carga psíquica. Os dados dessa pesquisa requerem informações futuras sobre os processos organizacionais e do modo de trabalho dessas categorias envolvidas.

\section{Conclusões}

O trabalho mostrou que, em 2002, os profissionais de enfermagem da rede básica de saúde de Campinas apresentaram valores de índices de freqüência e gravidade de absenteísmo acima da maioria dos estudos encontrados. Já o índice de freqüência foi maior no atual estudo que nos demais. A proporção de tempo perdido foi maior que a do único estudo que calculou esse índice ${ }^{23}$. Não há indícios nos dados desta pesquisa de que tais diferenças possam ser devidas à dupla jornada ou ao duplo emprego formal. Enfermeiros tiveram índice freqüência menor e gravidade maior, apontando para maior duração dos afastamentos dos enfermeiros em relação aos de auxiliares e técnicos.

Com relação aos índices de absenteísmo, é necessária a criação de consenso para uniformizar os cálculos de índices e tornar possíveis as comparações. A dificuldade encontrada neste trabalho foi de comparar os índices com autores que adotaram outras maneiras de calculá-los.

Como medida de curto prazo, a fim de diminuir a exposição aos riscos, propõese a diminuição das cargas de trabalho. Além disso, projetado para médio e longo prazo, sugere-se o aperfeiçoamento da política de saúde do trabalhador para os servidores da categoria.

\section{Referências}

1. Ferreira ABH. Novo Aurélio Século XXI: o dicionário da língua portuguesa. $3^{\mathrm{a}}$ ed. Rio de Janeiro: Nova Fronteira; 1999. p. 17.

2. Silva DMPP, Marziale, MHP. Absenteísmo de trabalhadores de enfermagem em um Hospital Universitário. Rev Latinoam Enfermagem 2000; 8(5): 44-51.

3. Jorge AL. Motivos que levam os trabalhadores de enfermagem ao absenteísmo. Acta Paul Enf 1995; 8(1): 39-46.

4. Robazzi MLCC, Paracchini AS, Gir E et al. Serviço de Enfermagem: um estudo sobre os absenteísmos. Rev Bras Saúde Ocupacional 1990; 18(69): 65-70.
5. Barboza DB, Soler ZASG. Afastamentos do trabalho na enfermagem: ocorrências com trabalhadores de um hospital de ensino. Rev Latinoam Enfermagem 2003; 11(2.): 177-83.

6. Echer IC, Moura GM, Magalhâes AM et al. Estudo do absenteísmo como variável no planejamento de recursos humanos. Rev Gaúcha de Enfermagem 1999; 20(2): 65-76.

7. Silva MP. Absenteísmo: grande problema das chefias de enfermagem. Rev Gaúcha de Enfermagem 1983; 4(1): 85-98.

8. Bulhões I. Riscos do trabalho de enfermagem. Rio de Janeiro: Folha Carioca; 1994. 221p. 
9. Silva VEF. O desgaste do trabalhador de enfermagem: relação trabalho de enfermagem e saúde do trabalhador [tese de doutoramento]. São Paulo: USP, 1996. 260p.

10. Reis RJ, LA Rocca PF, Silveira AM et al. Fatores relacionados ao absenteísmo por doença em profissionais de enfermagem. Rev Saúde Pública 2003; 37(5): 616-23.

11. Bridges S, Mumford K. Absenteeism in the UK: a comparison across genders. The Manchester School 2001; 69(3): 276-84.

12. Pines A, Skulkeo K, Pollak E. et al. Rates of sickness absenteeism among employees of a modern hospital: the role of demographic and occupational factors. Br J Ind Med 1985; 42: 326-35.

13. Johns G. How methodological diversity has improved our understanding of absenteeism from work. Human Resource Management Review 2003; 13: 157-84.

14. Oliveira SM. Absenteísmo em mulheres [Monografia]. Rio de Janeiro: Curso de Especialização em Medicina do Trabalho, Departamento de Planejamento em Saúde, Instituto de Saúde da Comunidade, Centro de Ciências Médicas, Universidade Federal Fluminense. Monografia de conclusão número 87 [mimeo], 06 de Agosto de 1988. $22 \mathrm{p}$.

15. Barmby T. Worker absenteeism: a discrete hazard model with bivariate heterogeneity. Labour Economics 2002; 9: 469-76.

16. Pauly MV, Nicholson S, Xu J. et al. A general model of the impact of absenteeism on employers and employees. Health Economics 2002; 11: p.221-31.

17. Barmby T, Stephan G. Worker absenteeism: why firm size may matter. The Manchester School 2000; 68(5): 568-77.

18. Alves M. Causas do Absenteísmo na Enfermagem: uma dimensão do sofrimento no trabalho [tese]. São Paulo: USP, 1996. 140p.

19. Fundación Europea para la Mejora de las Condiciones de Vida y de Trabajo. La prevención del absentismo en el lugar de trabajo [Resumen]. Luxemburgo: Oficina de Publicaciones Oficiales de las Comunidades Europeas, 1997. $42 \mathrm{p}$.
20. Reis P, Ribeiro PCL. Detecção de agravos à saúde relacionados com o trabalho e o uso gerencial da informação. In: Mendes R. Patologia do trabalho. $2^{\text {a }}$ ed. São Paulo: Atheneu, 2003. p. 319-22.

21. Correa Filho HR. Outra contribuição da epidemiologia. In: Buschinelli et al. Isto é trabalho de gente?. In: Vida, doença e trabalho no Brasil. São Paulo: Vozes, 1993. pp. 187-214.

22. Mendes R. Medicina do trabalho e doenças profissionais. São Paulo: Sarvier, 1980. p. 56-7.

23. Saroja KI, Ramphal KG, Kasmini K. et al. Trends in absenteeism rates following psychological intervention preliminary results. Singapore Med J 1999; 40(5): 349-351.

24. Quick TC, Lapertosa JB. Análise do absentismo em usina siderúrgica. Rev Bras Saúde Ocupacional 1982; 40(10): 62-7.

25. Meira JBB. Absentismo por enfermidade - sugestões para seu controle. Rev Bras Saúde Ocupacional 1982; 40(10): 68-76.

26. Greco RM. O trabalho de enfermagem na rede básica de saúde de Juiz de Fora: condições favoráveis e desfavoráveis [tese de doutoramento]. São Paulo: USP; 2001. p. 34-72.

27. Hamoui Y, Sirit Y, Bellorin M. Absentismo laboral del personal administrativo de una universidad pública venezoelana, 2000-2002. Salud de los Trabajadores 2005; 13(2):107-18.

28. Vasconcelos CBV, Alves CAS, Zago R, Gnoatto RM. Relação processo de trabalho e sofrimento mental: a percepção dos profissionais de enfermagem de Unidades Básicas de Saúde. Porto Alegre: Escola de Saúde Pública do Estado do Rio Grande do Sul; 2005. 62 p.

Recebido em: 29/01/07

Versão final reapresentada em: 13/07/07 Aprovado em: 30/07/07 
ANEXO/APPENDIX

Formulário de Levantamento de Dados

Número Formulário:

Centro de Saúde:

Nome:

Tempo de serviço na prefeitura de Campinas (em anos) :

Carga horária semanal:

Dias perdidos por licença para tratamento de saúde (LTS):

Dias previstos para serem trabalhados:

Horas perdidas por licença para tratamento de saúde (LTS):

Horas previstas para serem trabalhadas:

Licença para Tratamento de Saúde iniciadas no ano de 2002:

\begin{tabular}{|c|c|c|c|c|c|c|c|c|c|c|c|c|}
\hline & JAN & FEV & MAR & ABR & MAI & JUN & JUL & AGO & SET & OUT & NOV & DEZ \\
\hline 01 & & & & & & & & & & & & \\
\hline 02 & & & & & & & & & & & & \\
\hline 03 & & & & & & & & & & & & \\
\hline 04 & & & & & & & & & & & & \\
\hline 05 & & & & & & & & & & & & \\
\hline 06 & & & & & & & & & & & & \\
\hline 07 & & & & & & & & & & & & \\
\hline 08 & & & & & & & & & & & & \\
\hline 09 & & & & & & & & & & & & \\
\hline 10 & & & & & & & & & & & & \\
\hline 11 & & & & & & & & & & & & \\
\hline 12 & & & & & & & & & & & & \\
\hline 13 & & & & & & & & & & & & \\
\hline 14 & & & & & & & & & & & & \\
\hline 15 & & & & & & & & & & & & \\
\hline 16 & & & & & & & & & & & & \\
\hline 17 & & & & & & & & & & & & \\
\hline 18 & & & & & & & & & & & & \\
\hline 19 & & & & & & & & & & & & \\
\hline 20 & & & & & & & & & & & & \\
\hline 21 & & & & & & & & & & & & \\
\hline 22 & & & & & & & & & & & & \\
\hline 23 & & & & & & & & & & & & \\
\hline 24 & & & & & & & & & & & & \\
\hline 25 & & & & & & & & & & & & \\
\hline 26 & & & & & & & & & & & & \\
\hline 27 & & & & & & & & & & & & \\
\hline 28 & & & & & & & & & & & & \\
\hline 29 & & - & & & & & & & & & & \\
\hline 30 & & - & & & & & & & & & & \\
\hline 31 & & - & & - & & - & & & - & & - & \\
\hline
\end{tabular}

Legenda:

LTS: Licença para Tratamento de Saúde

Fi: Falta Injustificada

SAB: Sábado

DOM: Domingo

FER: Feriado 